\title{
BRCA2 gets protective at forks
}

The breast cancer type 2 susceptibility (BRCA2) protein, which is frequently mutated in hereditary breast cancer, is involved in the repair of DNA doublestrand breaks by homologous recombination (homology-directed repair (HDR)). Now, Schlacher et al. find that BRCA2 reduces genomic instability in an HDR-independent manner by preventing the nuclease meiotic recombination 11 (MRE11) from degrading stalled replication forks.

DNA replication forks can stall on exposure to drugs such as hydroxyurea, and, as the authors found, they are normally protected until replication can safely resume. However, they found that BRCA2-deficient cells are unable to protect stalled replication forks and, as a result, the

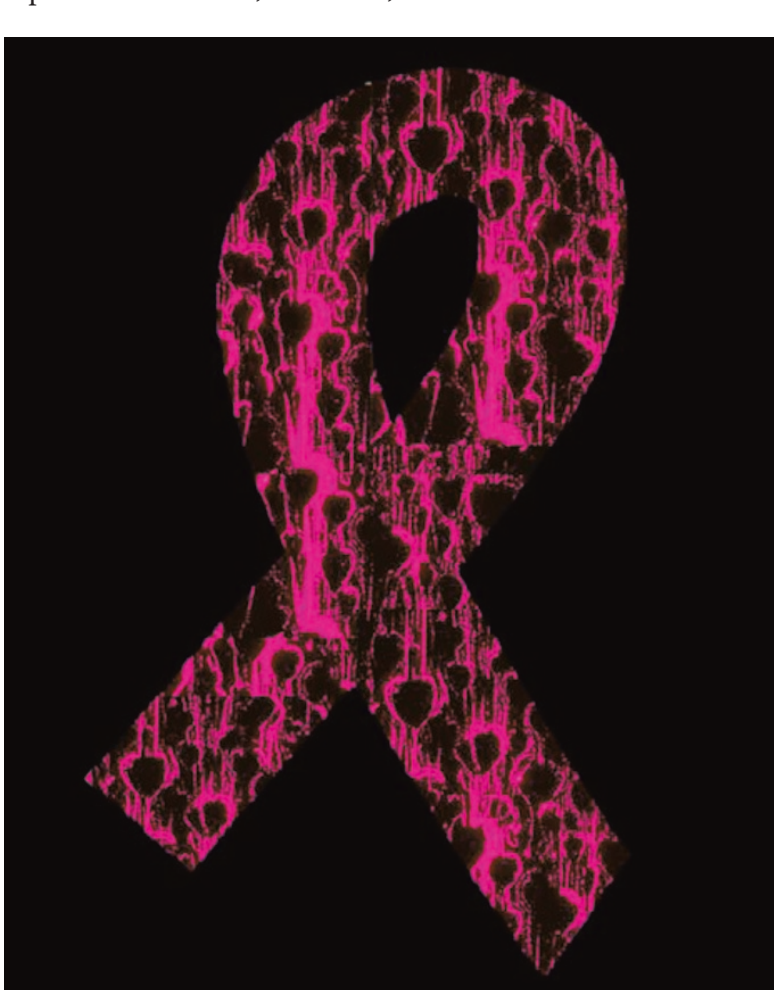
degradation.

The image shows a false-colour stain of DNA spreads. The technique allows the visualization of nascent replication tracts with single-molecule resolution. Image courtesy of K. Schlacher, Memorial Sloan-Kettering Cancer Center, USA. newly synthesized DNA is degraded. To show this, they used a DNA fibre assay in which DNA is pulse-labelled with nucleotides that stain fluorescent green or red before and after hydroxyurea treatment, respectively. This setup allows the stability of stalled replication forks to be inferred from the length of green-labelled DNA and the recovery of replication to be inferred from the length of

5
red-labelled DNA. In several mammalian cell types that are deficient for BRCA2, the authors found that the nascent green strands were significantly shorter; however, the recovery of replication after hydroxyurea treatment was largely unaffected. These results suggested that BRCA2 protects nascent DNA at stalled replication forks from degradation.

BRCA2 is a multidomain protein containing eight $\mathrm{BRC}$ repeats, which interact with the DNA repair protein RAD51, a DNA-binding domain and a carboxy-terminal region that interacts with RAD51 (known as C-ter). Deletion of the BRC repeats is embryonic-lethal owing to the requirement for normal HDR during embryogenesis. By contrast, BRCA2 mutations lacking the $\mathrm{C}$-ter survive early development but have an increased risk of tumorogenesis. Schlacher et al. found that BRCA2mutant cells lacking the DNA-binding domain (which are still competent for HDR) display no difference in replication fork stability; thus, it is unlikely that BRCA2 protects against degradation by directly binding DNA. However, cells containing Ser3291Ala-mutant BRCA2, which cannot associate with RAD51 at the C-ter, showed disrupted replication fork stability. Importantly, these cells are not deficient in HDR; thus, the roles of BRCA2 in HDR and in preventing the degradation of stalled replication forks are genetically distinguishable. Confirming the role of RAD51, the authors showed that expression of a peptide that blocks RAD51 binding to DNA caused degradation of nascent DNA.

Why is BRCA2 required to protect stalled replication forks? MRE11 is a nuclease that normally promotes DNA repair by processing DNA ends. As the kinetics of replication fork degradation were similar to the kinetics of MRE11 activity, the authors hypothesized that MRE11 might be responsible for fork degradation. Consistent with this, MRE11 inhibition protected against replication fork degradation in BRCA2-deficient cells. The authors propose a model in which BRCA2 stabilizes RAD51 filaments at stalled replication forks, which prevents degradation of nascent strands by MRE11.

The consequences of failure to protect stalled replication forks from degradation are severe, as the hydroxyurea-treated Ser3291Ala-mutant BRCA2-containing cells have a significantly higher number of chromosomal aberrations at metaphase than wild-type cells. This role of BRCA2 in protecting against genomic instability has important therapeutic implications. This is because cancers with BRCA2 mutations may not be killed by chemotherapies that stall replication; but instead, they may accumulate mutations at a faster rate, potentially enhancing tumorigenesis. Joanna E. Huddleston

ORIGINAL RESEARCH PAPER Schlacher, K. et al. Double-strand break repair-independent role for BRCA2 in blocking stalled replication fork degradation by MRE11. Cell 145, 529-542 (2011) FURTHER READING Branzei, D. \& Foiani, M. Maintaining genome stability at the replication fork. Nature Rev. Mol. Cell Biol. 11, 208-219 (2010)| Moynahan, M. E. \& Jasin, M. Mitotic homologous recombination maintains genomic stability and suppresses tumorigenesis. Nature Rev. Mol. Cell Biol. 11, 196-207 (2010) 